patients with pathological N2 disease was 806 days, with 30 day survival of $99 \%$ and 1 year survival $76 \%$.

Conclusions Lung cancer patients with stage IIIA disease make up a very small proportion of the overall lung cancer population. Only a small proportion of these patients receive surgery and there is significant discrepancy between the recorded pre and post operative nodal status. In patients with pathological confirmed N2 disease survival is similar to the 713 days reported in the Albain study. The automated collection of detailed radiotherapy/chemotherapy treatment data in future will allow a more reliable comparison between surgical and non-surgical treatments.

\section{S108 STATIN USE AND CIGARETTE SMOKING ARE ASSOCIATED WITH LOWER INCIDENCE OF RADIATION PNEUMONITIS}

${ }^{1} \mathrm{~L}$ Robinson, ${ }^{1} \mathrm{~A}$ Yeung, ${ }^{2} \mathrm{~T}$ Ali, ${ }^{2} \mathrm{SA}$ Worthy, ${ }^{3} \mathrm{PM}$ Mulvenna, ${ }^{3} \mathrm{M}$ Wilkinson, ${ }^{4} \mathrm{AJ}$ Simpson; ${ }^{1}$ Freeman Hospital, Newcastle Upon Tyne, United Kingdom; ${ }^{2}$ Royal Victoria Infirmary, Newcastle Upon Tyne, United Kingdom; ${ }^{3}$ Northern Centre for Cancer Care, Freeman Hospital, Newcastle Upon Tyne, United Kingdom; ${ }^{4}$ University of Newcastle Upon Tyne, Newcastle Upon Tyne, United Kingdom

\subsection{6/thoraxjnl-2013-204457.115}

Background Three months after radical radiotherapy for lung cancer, $50-60 \%$ of patients have radiation pneumonitis (RP) on CT thorax. Our aim was to assess the clinical and dosimetric factors associated with radiologically-defined RP. Our primary endpoint was the development of new infiltrates on CT thorax at 3 months following radiotherapy.

Methods 161 patients with lung cancer were referred for radical radiotherapy during 2009-2010. Exclusion criteria were previous thoracic radiotherapy or surgery, palliative radiotherapy, or missing dosimetric or CT data.

Information on medical history, lung function and date of death were taken retrospectively from electronic notes. Dosimetric parameters V20-Lung (percentage normal lung exposed to more than 20Gy), V5-Lung and Mean Lung Dose were derived from treatment planning dose-volume histograms. Development of RP was defined as an increase in the percentage lung volume occupied by consolidation or ground glass on post-radiotherapy CT. Student's t-test and Fisher's Exact Test were used to define variables which were associated with RP prior to logistic regression analysis.

Results 98 cases were included in analysis. $86 \%$ had non-small cell lung cancer, $44 \%$ had chronic obstructive pulmonary disease (COPD), and 27\% smoked. 49/98 (50\%) patients developed RP on CT at median 90 days post-radiotherapy.

The factors which had a significant positive correlation with RP on univariate analysis were V20-lung, V5-lung and MLD: these were best represented using V20-Lung $\geq 22 \%$. Current smoking, poor performance status and having COPD had a significant inverse correlation with RP. Use of statins or inhaled Long Acting $\beta 2$ Agonists, and the presence of moderate-severe radiological emphysema also approached significance: these were included in regression analysis.

After logistic regression, the factors which had a significant correlation with RP were V20 $\geq 22 \%$ (OR 6.45, 95\%CI 2.2218.08), current smoking (OR $0.23,95 \% \mathrm{CI} 0.07-0.79$ ), and statin use (OR 0.30, 95\%CI 0.102-0.863).

Neither RP nor any other variable was associated with postradiotherapy mortality.

Conclusions This study confirms that V20 $\geq 22 \%$ is associated with the radiological development of RP. In addition, patients who smoked, and those taking statins were significantly less likely to develop RP. A potential role for statins in modifying radiotherapy side effects deserves further attention.

\section{S109 THE INTRODUCTION OF STEREOTACTIC ABLATIVE RADIOTHERAPY INCREASES OVERALL RADICAL TREATMENT RATES FOR STAGE I LUNG CANCER BUT DOES NOT REDUCE SURGICAL RESECTION RATES-A TWO CENTRE STUDY}

${ }^{1} \mathrm{~L}$ Cheyne, ${ }^{2} \mathrm{G}$ Esterbrook, 'S Viadyanathan, ${ }^{1} \mathrm{R}$ Milton, ${ }^{2} \mathrm{G}$ Smith, ${ }^{2} \mathrm{P}$ Blaxill, ${ }^{1} \mathrm{~K}$ Clarke, ${ }^{1} \mathrm{M}$ Snee, ${ }^{1} \mathrm{~K}$ Franks, ${ }^{1} \mathrm{MEJ}$ Callister; ${ }^{1}$ The Leeds Teaching Hospitals NHS Trust, Leeds, UK; ${ }^{2}$ The Mid Yorkshire Hospitals NHS Trust, Wakefield, UK

\subsection{6/thoraxjn-2013-204457.116}

Background Stereotactic ablative radiotherapy (SABR) is a new treatment option for peripheral stage I lung cancer in patients unfit for surgical resection. SABR was introduced to Leeds Teaching Hospitals (LTHT) and Mid Yorkshire Hospitals (MYH) in May 2009. We sought to establish what effect the introduction of SABR had on surgical resection rates for stage I lung cancer, and compared clinical characteristics of patients receiving surgery, SABR, conventional radical radiotherapy (RRT) and best supportive care.

Methods All patients diagnosed with stage I lung cancer from 2008 to 2011 were analysed for treatment modality, performance status (PS) and lung function.

Results 565 stage I patients were studied and treatment rates over the 4 year period are shown below. The proportion of patients receiving SABR rates rose from $0 \%$ in 2008 to $26.1 \%$ in 2011. Surgical resection rates remained largely unchanged, but there was a reduction in the proportion of patients receiving best supportive care from $32.6 \%$ in 2008 to $13.7 \%$ in 2011 . Overall radical treatment rates for the four years were $60 \%$, $70.7 \%, 68 \%$ and $85 \%$ for $2008-2011$ respectively.

The proportion of patients with PS $0-1$ were as follows: surgery $88 \%$, SABR $39 \%$, RRT $38 \%$ and BSC $13 \%$. FEV 1 (l) (mean $\%$ predicted, 95\% CI) was significantly higher in patients receiving surgery $(80.1,77.3-82.9)$ compared to those patients receiving SABR $(62.1,56.0-68.3, \mathrm{p}<0.001$ vs surgery), RRT $(62.7,54.2-$ $71.3, \mathrm{p}<0.001$ vs surgery) and BSC $(56.4,49.8-63.0, \mathrm{p}<0.001$ vs surgery). Similarly gas transfer was significantly higher in the surgical patients compared to the other three groups.

For stage I lung cancer patients over the age of 75 , the proportion of patients SABR rose from $0 \%$ in 2008 to $32.1 \%$ in 2011. Overall numbers of patients aged over 75 receiving BSC decreased over the four years; $49 \%, 45.5 \%, 38.6 \%$ and $24.4 \%$ for 2008-2011 respectively.

Conclusion The introduction of SABR has led to a significant increase in overall radical treatment rates for patients with stage I lung cancer, without resulting in a sustained reduction in surgical resection rates. Patients undergoing SABR and RRT have worse lung function and performance status than those undergoing surgery.

\section{S110 EXPERIENCE WITH SUSPECTED CANCER REFERRALS FROM THE UK LUNG SCREEN TRIAL}

${ }^{1} \mathrm{G}$ Jones, ${ }^{1} \mathrm{D}$ Komrower, ${ }^{1} \mathrm{M}$ Murthy, ${ }^{1} \mathrm{~N}$ Hunt, ${ }^{1} \mathrm{~J}$ Holemans, ${ }^{2} \mathrm{~J}$ Field, ${ }^{1} \mathrm{M}$ Ledson, ${ }^{1} \mathrm{M}$ Walshaw; 'Liverpool Heart \& Chest Hospital, Liverpool, United Kingdom; ${ }^{2}$ University of Liverpool, Liverpool, United Kingdom

10.1136/thoraxjnl-2013-204457.117 
Introduction Early diagnosis of lung cancer improves survival and strategies to facilitate this include screening high risk populations. The ongoing UK Lung Screen Trial (UKLS) is investigating this with low-dose CT scans, and positive screens are referred to clinical services for further investigation and treatment. We report the outcome for such cases referred to our large lung cancer unit.

Methods The UKLS reporting radiologists code scans according to their abnormality: local patients with features suspicious for malignancy (category 4) are referred to our MDT, where following counselling they undergo appropriate investigation.

Results We have received 37 referrals (mean age68 [range 6175], median performance status 1 , mean FEV1 $78 \%$ predicted [33-107], 27 male) during the first 14 months of the trial.

In 23 cases $(62 \%)$ subsequent investigation confirmed malignancy (11 adenocarcinoma, 8 squamous cell carcinoma, 3 small cell carcinoma, 1 mesothelioma). In 14 cases where malignancy was not confirmed all had been referred for additional investigations (including 3 PET-CT scans, 5 bronchoscopies and 8 CT scans): 1 patient underwent wedge resection of a PET positive lesion (granulomatous process) and 13 remain under follow-up for nodule surveillance.

Twenty of those with malignancy $(87 \%)$ were potentially operable (median stage 1A, mean age 68 [range 61-74], median performance status 1, mean FEV1 80\% predicted [33-107], 13 male), including 2 with limited stage small cell carcinoma. Overall, 19 patients were treated by lobectomy with curative intent. The remaining 4 cases all received oncological management.

Discussion The UKLS is the first large randomised controlled trial to assess a CT-based screening protocol for lung cancer in the United Kingdom. We have shown that the majority of referrals from this programme were confirmed as cases of lung cancer of which nearly all were operable. Such screening programmes will help us improve the prognosis in this previously late-diagnosed and often incurable disease.

\section{COPD: mechanisms of host defence}

\section{S111 HUMAN RHINOVIRUS INFECTION AND EXACERBATION FREQUENCY AT COPD EXACERBATION}

SN George, ARC Patel, AJ Mackay, R Singh, RJ Sapsford, GC Donaldson, JA Wedzicha; Centre for Respiratory Medicine, UCL, London, UK

\subsection{6/thoraxjnl-2013-204457.118}

Introduction Viral infections are associated with more severe COPD exacerbations in terms of greater burden of symptoms, resulting in longer recovery times and a greater likelihood of hospitalisation (Seemungal et al. 2000). Human rhinoviruses (HRV) are the main aetiological agents of virus-associated COPD exacerbations; however the association of HRV infection and exacerbation frequency has not been fully investigated. We aimed to examine the relationship between HRV presence and load, and the number of exacerbations patients experienced per year.

Methods London COPD cohort patients recorded any new or increased respiratory symptoms on daily diary cards and contacted the clinical team when symptoms worsened. Exacerbations were defined using our usual symptomatic criteria; an increase in respiratory symptoms for two consecutive days, with at least one symptom being major (dyspnoea, sputum purulence or volume). Reverse-transcription quantitative PCR was used to detect HRV prevalence and load in sputum samples collected at exacerbation presentation (median 2 days after exacerbation onset).

Results Patients positive for HRV $(n=42)$ had significantly more exacerbations per year than those without $\operatorname{HRV}(\mathrm{n}=31)$; the median (IQR) number of exacerbations per year in those with HRV infection was $3.01(2.02-5.30)$ which was significantly greater than those without HRV infection 2.51 (2.00-3.51); $\mathrm{p}=0.038$ (Figure 1A). At exacerbation, a higher HRV load significantly correlated with the number of exacerbations patients experienced per year; $r=0.145 ; \mathrm{p}=0.024$ (Figure 1B).

Conclusion Patients with positive HRV infection at the time of exacerbation had experienced more exacerbations per year than those who did not have HRV. In patients with a higher exacerbation frequency, the HRV load at exacerbation was greater suggesting that patients with a history of frequent exacerbations are more susceptible to viral infection. This susceptibility may provide the mechanisms for the development of the frequent exacerbator phenotype. Thus these findings emphasise the importance of preventing viral infections and exacerbations in COPD patients.
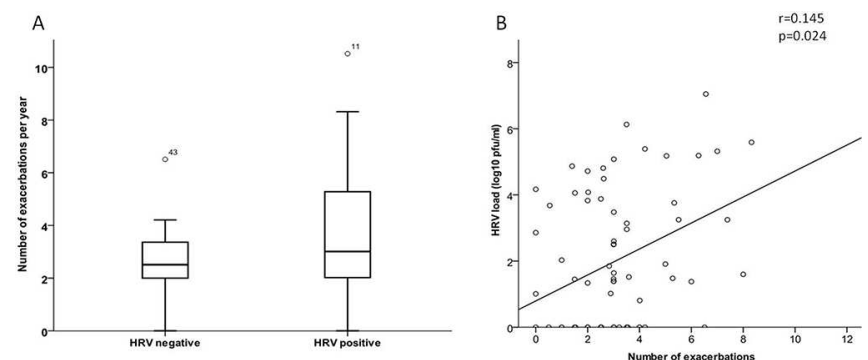

Abstract S111 Figure 1. (A) The number of exacerbations per year in patients with $\mathrm{HRV}(\mathrm{n}=42)$ was significantly higher than in those without HRV $(n=31), p=0.038$. (B) There was an association between the number of exacerbations had by patients per year and the HRV load $(r=0.145 ; p=0.024)$.

\section{S112 HDAC ACTIVITY IN MACROPHAGES IN EXPERIMENTAL RHINOVIRUS INFECTION IN COPD}

J Footitt, P Mallia, A Durham, MB Trujillo-Torralbo, A Telcian, T Kebadze, J Aniscenko, S Essilfie-Quaye, K Ito, PJ Barnes, S Elkin, OM Kon, I Adcock, SL Johnston; Imperial College, London, UK

\subsection{6/thoraxjnl-2013-204457.119}

Introduction and Objectives Acute exacerbations are a major cause of morbidity and mortality in COPD and current treatments are not very effective. Histone deacetylase 2 (HDAC2) is deficient in stable COPD and is likely to be a mechanism of corticosteroid resistance. It is not known whether impaired HDAC2 activity is an important mechanism in COPD exacerbations.

Methods 9 subjects with GOLD stage II COPD, 10 smokers and 11 non-smokers were infected with rhinovirus 16. Macropahges from induced sputum and bronchoalveolar lavage (BAL) were collected before and following rhinovirus infection and HDAC2 activity measured. Virus load and inflammatory markers were measured in sputum supernantants.

Results At baseline there were no differences in HDAC2 activity in sputum or BAL macrophages between the groups. Following infection HDAC2 activity in the smoking controls and nonsmoking controls did not change significantly from baseline (Figure 1). In the COPD subjects there was a trend towards reduced HDAC2 activity in both sputum (ANOVA P $=0.064$ ) and BAL macrophages (Paired t test $\mathrm{P}=0.098$ ). Sputum HDAC activity 